\title{
The perception of self-produced sensory stimuli in patients with auditory hallucinations and passivity experiences: evidence for a breakdown in self-monitoring
}

\author{
S.-J. BLAKEMORE, ${ }^{1}$ J. SMITH, R. STEEL, E. C. JOHNSTONE AND C. D. FRITH \\ From the Wellcome Department of Cognitive Neurology, Institute of Neurology, London; and \\ Department of Psychiatry, Royal Edinburgh Hospital, Edinburgh
}

\begin{abstract}
Background. To test the hypothesis that certain psychotic symptomatology is due to a defect in selfmonitoring, we investigated the ability of groups of psychiatric patients to differentiate perceptually between self-produced and externally produced tactile stimuli.

Methods. Responses to tactile stimulation were assessed in three groups of subjects: schizophrenic patients; patients with bipolar affective disorder or depression; and normal control subjects. Within the psychiatric groups subjects were divided on the basis of the presence or absence of auditory hallucinations and/or passivity experiences. The subjects were asked to rate the perception of a tactile sensation on the palm of their left hand. The tactile stimulation was either self-produced by movement of the subject's right hand or externally produced by the experimenter.
\end{abstract}

Results. Normal control subjects and those psychiatric patients with neither auditory hallucinations nor passivity phenomena experienced self-produced stimuli as less intense, tickly and pleasant than identical, externally produced tactile stimuli. In contrast, psychiatric patients with these symptoms did not show a decrease in their perceptual ratings for tactile stimuli produced by themselves as compared with those produced by the experimenter. This failure to show a difference in perception between self-produced and externally produced stimuli appears to relate to the presence of auditory hallucinations and/or passivity experiences rather than to the diagnosis of schizophrenia.

Conclusions. We propose that auditory hallucinations and passivity experiences are associated with an abnormality in the self-monitoring mechanism that normally allows us to distinguish selfproduced from externally produced sensations.

\section{INTRODUCTION}

Humans can readily detect and distinguish whether sensory signals are the result of selfgenerated actions or other environmental events. It has been proposed that knowledge of our intentions or motor commands is used to distinguish the sensory consequences of our own actions from externally produced sensations

${ }^{1}$ Address for correspondence: Ms Sarah-Jayne Blakemore, Wellcome Department of Cognitive Neurology, Institute of Neurology, 12 Queen Square, London WC1N 3BG.
(Frith, 1992; Wolpert et al. 1995; Decety, 1996; Jeannerod, 1997; Wolpert, 1997). In order to achieve this, some kind of central monitor (Frith, 1992) or internal 'forward model' (Fig. 1) (Wolpert et al. 1995; Wolpert, 1997) has been postulated. Forward models capture the forward or causal relationship between actions, as signalled by an efference copy of the motor command (Von Holst, 1954), and the sensory outcome. It is proposed that efference copy signals are used to make a prediction of the sensory consequences (corollary discharge; Sperry, 1950) of the motor act and this prediction 


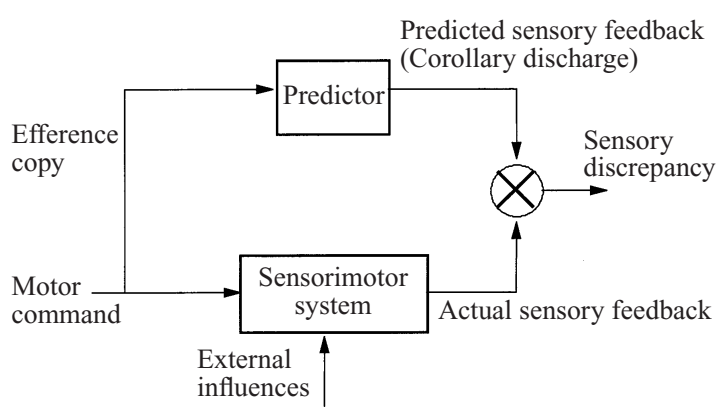

FIG. 1. A model for predicting the sensory consequences of a movement, in the present study when the right hand is causing the tactile sensation on the left palm. An internal forward model makes predictions of the sensory feedback based on the motor commands sent to the right hand. These sensory predictions are then compared to the actual sensory feedback. The lower the sensory discrepancy resulting from this comparison the greater is the attenuation of tactile sensation. In our study, when the tactile stimulation is self-produced the model correctly predicts the sensory consequences of the movement so no sensory discrepancy ensues between the predicted and actual sensory information. In this case the motor command to the right hand can be used to attenuate the sensation on the left palm. When the sensory feedback deviates from the prediction of the model (when tactile stimulation is externally produced) the level of sensory discrepancy between predicted and actual sensory feedback is increased. This leads to a decrease in the amount of attenuation possible and a relative increase in the intensity of sensation experienced on the left palm.

is then compared with the actual consequences of such an act. If the prediction matches the actual sensory consequences then the sensation is likely to be self-generated.

These mechanisms have been the subject of much investigation, mainly in the oculomotor domain. However, it appears that sensory predictions produced in conjunction with motor commands are not restricted to eye movements, but also provide perceptual stability in the context of all self-produced actions. Although subject to debate (see Gallagher, 1999), we argue that our ability to monitor, and recognize as our own, self-generated limb movements, touch, speech and thoughts suggests the existence of a more general mechanism (Frith, 1992). Selfproduced sensations can be correctly predicted on the basis of the motor command, and there will therefore be little or no difference between predicted and actual sensory feedback. As the difference increases so does the likelihood that the sensation is externally produced. By using such a system it is possible to cancel out the effects of sensation induced by self-motion and thereby distinguish sensory events due to selfproduced motion from the sensory feedback caused by the environment. The perceptual attenuation of tactile sensation associated with self-produced actions is well-documented in humans (Angel \& Malenka, 1982; Chapman et al. 1987; Milne et al. 1988; Collins et al. 1998). An example of such attenuated perception is the phenomenon that people cannot tickle themselves (Weiskrantz et al. 1971). We have demonstrated that self-produced and externally produced tactile sensations are perceived differently in normal subjects (Blakemore et al. 1999). Normal subjects consistently rated a selfproduced tactile sensation on their right palm as being significantly less 'tickly', 'intense' and 'pleasant' than an identical stimulus produced externally. We suggested that this is because self-produced tactile stimulation can be accurately predicted on the basis of the motor command producing it using a forward model of the motor system.

Frith (1992) proposed that a defect in central self-monitoring might underlie auditory hallucinations and passivity phenomena experienced by people with schizophrenia. Auditory hallucinations are common in schizophrenia, and normally consist of hearing spoken speech or voices (Hoffman, 1986; Johnstone, 1991). Certain types of auditory hallucinations are included as 'first rank' features in schizophrenia, features that have been regarded as pathognomic of the disorder in most circumstances (Schneider, 1959). These features have much in common with the 'nuclear syndrome of schizophrenia' described by the PSE/CATEGO system (Wing et al. 1974). Passivity experiences or delusions of alien control are further 'first rank' features in schizophrenia (Schneider, 1959). The essence of this symptom is that the subject experiences his or her will as replaced by that of some other force or agency (Wing et al. 1974): 'My fingers pick up the pen, but I don't control them. What they do is nothing to do with me ... The force moved my lips. I began to speak. The words were made for me' (Mellors, 1970).

Frith (1992) has suggested that these abnormal experiences arise through a lack of awareness of intended actions. Such an impairment might cause thoughts or actions to become isolated from the sense of will normally associated with them. This would result in the interpretation of internally generated voices or thoughts as external voices (auditory hallucinations and 
thought insertion) and of one's own movements and speech as externally caused (passivity or delusions of control). We have suggested that the experience of passivity arises from a lack of awareness of the predicted limb position based on the forward model (Frith et al. 2000). Thus, the patient is aware of the intention to move and of the movement having occurred, but is not aware of having initiated the movement. It is as if the movement, although intended, has been initiated by some external force. In a variation on this theme, Spence (1996) has suggested that the problem is to do with the timing of awareness. The awareness of the actual outcome of the movement precedes the awareness of the predicted outcome, which is contrary to the normal experience of our own agency.

There is nothing obviously abnormal in the motor control of these patients. However, there are subtle problems consistent with a lack of awareness of predicted actions. Two experiments, in which subjects had to correct their errors very rapidly in the absence of visual feedback, found evidence that central monitoring is faulty in schizophrenia (Malenka et al. 1982; Frith \& Done, 1989). Normal control subjects were adept at this task, suggesting that they monitor the response intended (via corollary discharge) and do not need to wait for external feedback about the response that actually occurred. Failure to correct their errors in the absence of feedback was characteristic of schizophrenic patients with passivity symptoms in the study by Frith \& Done (1989).

A breakdown in self-monitoring might result in failure in the perceptual modulation of selfproduced stimuli that is seen in normal subjects (Weiskrantz et al. 1971; Blakemore et al. 1999). In the present study, we sought to evaluate whether patients with auditory hallucinations and passivity experiences differentiate perceptually between tactile stimuli that are selfproduced and externally produced. Patients with a diagnosis of schizophrenia, bipolar affective disorder or depression were divided into two groups according to the presence or absence of auditory hallucinations and passivity experiences. Subjects were asked to rate a tactile sensation (caused by a piece of soft foam) on the palm of their left hand that was produced either by movement of their right hand or by the experimenter. Based on the theory outlined above (Frith, 1992; Frith et al. 2000) we predicted that patients with auditory hallucinations and passivity experiences would be abnormally aware of the sensory consequences of their own movements, and thus would not show the normal attenuation of self-produced relative to externally produced sensations.

\section{METHOD}

\section{Subjects}

Patients with a diagnosis of schizophrenia $(N=23 ; 16$ males; mean age $33 \pm 1.8$ years; 22 were right-handed), affective disorder (bipolar or depression; $N=18$; nine males; mean age $39 \pm 2 \cdot 8$ years; 16 were right-handed) and a group of normal control subjects $(N=15$; nine males; mean age $32 \pm 2.07$ years; all were righthanded) gave informed consent to take part in the study. All patients were diagnosed by clinical interview (by J.S.) using the ICD-10 (International Classification of Diseases; WHO, 1992). The study was approved by the Psychiatry and Clinical Psychology Ethics Subcommittee of the Lothian Research Ethics Committee.

\section{Division of patient groups}

Patients were divided into two groups in terms of the presence or absence of auditory hallucinations and/or passivity experiences, according to a questionnaire given to each subject by R.S. before the experiment. This questionnaire assessed general, background and clinical information. Subjects were asked about their symptomatology, specifically whether they had experienced auditory hallucinations, thought interference or passivity phenomena within the past 6 weeks. The questionnaire was performed on, and externally validated (by S.-J. B. and R.S.) for all (schizophrenic, manic and depressed) patients and normal control subjects (unpublished data).

The patients with a diagnosis of schizophrenia were also assessed by the PANSS interview (Kay et al. 1987) conducted by J.S. with video monitoring. A score of 3 ('mild') on the Hallucinatory Behaviour scale of the PANSS was taken to indicate the presence of auditory hallucinations. Patients who scored 1 ('absent') or 2 ('minimal') on this scale were classified as not experiencing auditory hallucinations. The average score on the Hallucinatory Behaviour 
scales was $1 \cdot 1$ for the group of patients without auditory hallucinations and 3.9 for the group classified as experiencing auditory hallucinations. There was $100 \%$ concordance between classification based on symptom ratings from the questionnaire and the PANSS ratings for patients with PANSS results. PANSS results were only available for patients with a diagnosis of schizophrenia.

According to the questionnaire a total of 17 (13 schizophrenic and four affective) patients had experienced auditory hallucinations and/or passivity phenomena within the past 6 weeks. While all 17 subjects had experienced auditory hallucinations, six had also experienced passivity phenomena within the past 6 weeks. Twentyfour (10 schizophrenic and 14 affective) patients had not experienced either of these symptoms within the past 6 weeks.

For an overall picture of psychopathology within the patients with schizophrenia, the PANSS scales were divided into three categories corresponding to Reality Distortion, Disorganization and Psychomotor Poverty, and the average score on each of these scales was calculated for the schizophrenic patients. The average scores were as follows: Reality Distortion 2.9; Disorganization 2.0; Psychomotor Poverty $2 \cdot 2$.

\section{Procedure}

The tactile stimulus device consisted of a piece of soft foam attached to a plastic rod (length $70 \mathrm{~cm}$ ) which could pivot about its centre (Fig. 2). The rod was situated inside a plastic box attached to a perspex sheet, which was attached to a table. Subjects sat facing the table, with their left arm perpendicular to the table and secured to the perspex sheet. The piece of foam touched the subject's left palm, and could be moved by the movement of the rod. The rod could be moved either by the subject using their right hand or, from the other end of the rod, by the experimenter (S.-J.B.). The rotation of the rod was mechanically limited to vertical sinusoidal movements of amplitude $1.5 \mathrm{~cm}$. Subjects were instructed to move the rod up and down to its full extent at a frequency of $2 \mathrm{~Hz}$ and practised beforehand to ensure that they could reliably generate the desired movements. This took between 1 and $4 \mathrm{~min}$ for each subject. The frequency of stimulus movement in each trial was recorded by the experimenter (S.-J.B.) throughout the experiment.

\section{Self-generated tactile stimulation}

Subjects were required to make vertical sinusoidal movements of the rod with their right hand. This movement produced tactile stimulation on the palm on their left hand.

\section{Externally produced tactile stimulation}

The experimenter moved the tactile stimulus sinusoidally across the subject's left palm, while the subject rested their right hand.

Each trial lasted $10 \mathrm{~s}$. There were 10 repetitions of each condition for each subject, thus each subject underwent a total of 20 trials. The condition order was alternated. Before the experiment the apparatus was explained to each subject. They were told that the piece of foam would touch their hand in each condition, and that on half the trials the foam would be moved by themselves, and in the other half by the experimenter.

\section{Rating scale}

After each trial subjects were instructed to rate the sensation on their palm on a scale from 0 (not at all) to 10 (extremely) 'Intense', 'Painful', 'Tickly', 'Pleasant' and 'Irritating'. Subjects were told that a rating of zero indicated a neutral perception for that particular scale. Subjects were told that it was important to maintain the same scale all the way through the experiment and that the emphasis was on the relative values of their judgements, not the absolute values.

\section{Sandpaper control trials}

In order to obtain an objective assessment of the ability of subjects to rate a tactile sensation, subjects were asked to rate the roughness of four grades of sandpaper (grade numbers 80, 120, $240,460)$. The four different samples of sandpaper were attached to a sheet of card. Subjects were asked to close their eyes, move the fingers of their left hand over one piece of sandpaper and rate it in terms of its roughness on a scale from 0 (not at all rough) to 10 (extremely rough). The four samples of sandpaper were presented to the subjects in a randomized order. Subjects were excluded from the analysis if there was no difference between their ratings for the 


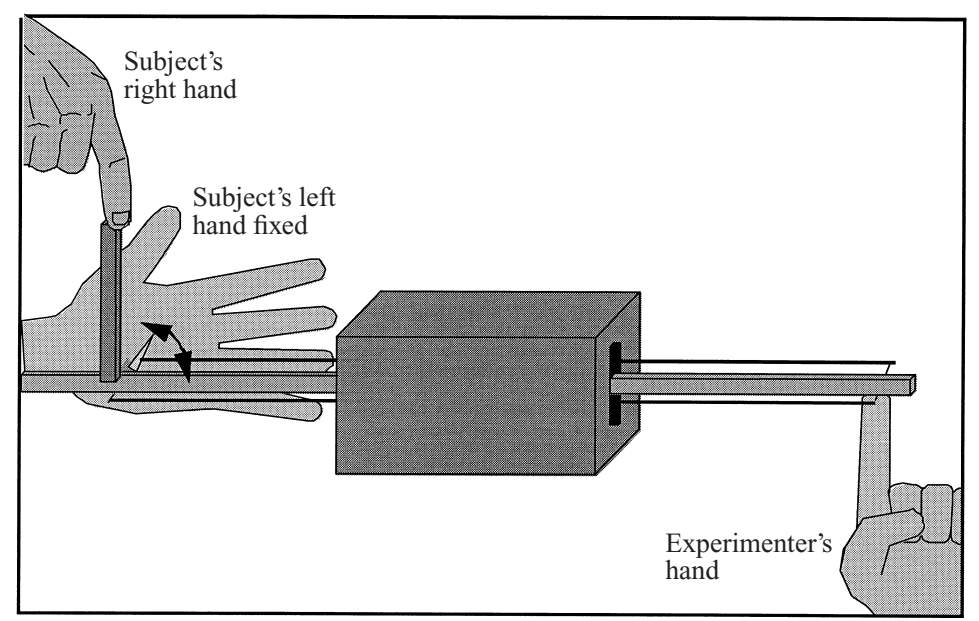

FIG. 2. Diagram of experimental set-up. The tactile stimulus device consisted of a piece of soft foam attached to a plastic rod (length $70 \mathrm{~cm}$ ) which could pivot about its centre. The rotation of the rod was mechanically limited to vertical movements of amplitude $1.5 \mathrm{~cm}$. The rod could be moved either by the subject using their right hand or, from the other end, by the experimenter. Movement of the rod caused movement of the piece of soft foam, which made light contact with the subject's left palm. See text for details.

roughness of the four grades of sandpaper, or if their ordering was wrong for more than two grades.

\section{Data analysis}

Analyses were performed on the data to test the hypothesis that the presence of auditory hallucinations and/or passivity symptoms has a significant effect on the perception of selfproduced stimuli. We predicted that patients with auditory hallucinations and/or passivity phenomena would show no significant difference between their perceptual ratings in the two tactile stimulation conditions. In contrast, we predicted that the patients without these symptoms and normal control subjects would show a significant difference between their ratings in the two conditions, rating self-produced tactile stimulation as less intense than externally produced stimulation.

Since each subject used their own subjective rating scale, the ratings need not conform to a normal distribution. We therefore used Wilcoxon non-parametric matched pairs signed ranks test (Howell, 1997) to examine the difference between conditions for each scale within each subject group. Results were taken as significant if $P<0.05$.

\section{RESULTS}

\section{Sandpaper control trials}

All except three subjects performed the experiment satisfactorily and rated the four grades of sandpaper in the correct order of roughness. Two of the three subjects who failed the sandpaper control test had diagnoses of schizophrenia and had experienced auditory hallucinations and/or passivity phenomena within the past 6 weeks. The third patient who failed the sandpaper control test had a diagnosis of bipolar disorder, and had not experienced auditory hallucinations and/or passivity phenomena within the past 6 weeks. All three subjects rated all four grades of sandpaper as equally rough. Their perceptual ratings data were therefore excluded from further analysis.

\section{Perceptual ratings analysis}

After practice each subject was able to produce the desired movement of the left hand with reasonable accuracy. The scales 'Painful' and 'Irritating' both received few positive ratings, and were therefore excluded from the analysis.

The results of the Wilcoxon non-parametric ranking test demonstrated that auditory hallucinations and/or passivity symptoms had a sig- 
nificant effect on perceptual ratings in the two conditions.

\section{Patients with auditory hallucinations and/or passivity experiences $(N=15)$}

There was no significant difference between selfproduced and externally produced tactile conditions for the intense $(Z=-1.54 ; P=0 \cdot 12)$, tickly $(Z=-1.88 ; \quad P=0.06)$ and pleasant $(Z=-1 \cdot 08 ; P=0 \cdot 28)$ ratings in patients with auditory hallucinations and/or passivity experiences.

\section{Patients without auditory hallucinations and/or passivity experiences $(N=23)$}

Patients without auditory hallucinations and/or passivity experiences rated self-produced tactile stimuli as significantly less intense $(Z=-4 \cdot 24$; $P<0.0001)$, tickly $(Z=-5 \cdot 10 ; P<0 \cdot 0001)$ and pleasant $(Z=-5.02 ; P<0.0001)$ than the externally produced tactile stimuli.

\section{Normal control subjects $(N=15)$}

The normal control subjects rated self-produced tactile stimuli as significantly intense $(Z=$ $-2.66 ; P<0.01)$, tickly $(Z=-3.10 ; P<0.005)$ and pleasant $(Z=-5.54 ; P<0.0001)$ than the externally produced tactile stimuli.

Because the results were similar for the 'Tickly', 'Intense' and 'Pleasant' rating scales, the average ratings from these three scales were combined to form one perceptual rating for each condition for each subject. The mean ratings for the combined perceptual scale for the selfproduced and the externally produced tactile stimulation conditions were $2 \cdot 10$ and 2.60 respectively for normal control subjects; 2.68 and $3 \cdot 15$ respectively for patients without auditory hallucinations and/or passivity phenomena; and 3.60 and 3.63 respectively for patients with these symptoms.

The difference between this combined perceptual rating in each condition (Self-produced rating-Externally produced rating) was calculated for each subject, and these mean difference ratings were used in the subsequent analysis (Fig. 3). Because these scores were not appropriately distributed we examined the differences between the groups in terms of the number of subjects who showed the 'normal' pattern of responding. We compared the number of subjects showing the 'normal' response (a

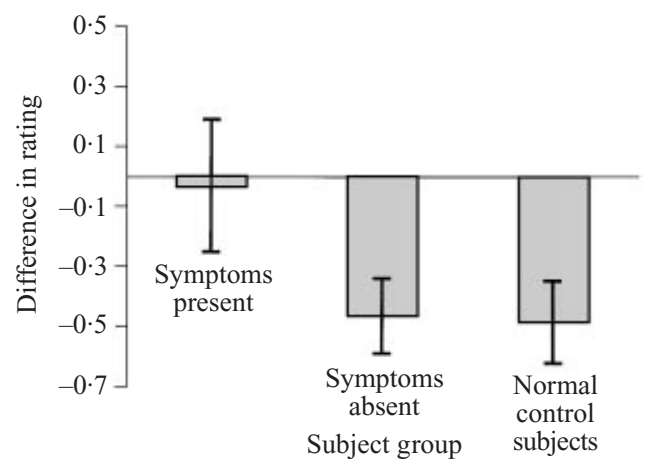

FIG. 3. Graph showing mean rating differences between selfproduced and externally produced tactile stimulation conditions for the three subject groups: patients with auditory hallucinations and/or passivity experiences, patients without these symptoms, and normal control subjects. There was no significant difference between the perceptual ratings in these two conditions of patients with auditory hallucinations and/or passivity experiences, hence the mean rating difference was close to zero. In contrast, there was a significant difference between the perceptual ratings in the two conditions of patients without with these symptoms and of normal control subjects: both groups rated self-produced stimulation as significantly less tickly, intense and pleasant than externally produced stimulation.

higher combined perceptual rating for externally produced tactile stimuli than for self-produced tactile stimuli) and the number subjects not showing this normal response in each subject group. Overall comparison of the three groups revealed a marginally significant difference $\left(\chi^{2}(2)=4 \cdot 74, \quad P<0 \cdot 09\right)$. However, direct examination of our prediction showed that significantly fewer patients with auditory hallucinations and/or passivity phenomena showed the normal response than patients without these symptoms and normal control subjects $\left(\chi^{2}(1)=\right.$ $4 \cdot 33, P<0.05)$.

\section{DISCUSSION}

The present study sought to investigate how patients with auditory hallucinations and/or passivity experiences perceive the sensory consequences of their own actions. We demonstrate that perception is not modulated by selfgenerated actions in patients with auditory hallucinations and passivity experiences. Patients without these symptoms and normal control subjects consistently rated a selfproduced tactile sensation as being less intense, tickly and pleasant than when the sensation was externally produced. In contrast patients with these symptoms did not show a decrease in their 
perceptual ratings for tactile stimuli produced by themselves as compared to those produced by the experimenter (Fig. 3).

We have previously shown that normal control subjects perceive self-produced tactile stimuli as less intense, tickly and pleasant than externally produced tactile stimuli (Blakemore et al. 1999), supporting and expanding on a study by Weiskrantz et al. (1971). We interpreted these results in terms of sensory predictions made by a forward model of the motor system (Fig. 1) (Wolpert, 1997). In terms of the current study, on the basis of the efference copy produced in parallel with the motor command, the forward model predicts the sensory consequences of the movement of the right hand. These sensory predictions are compared to the actual sensory feedback from the movement, and are used to cancel the tactile consequences of the movement. Self-produced sensations can be correctly predicted on the basis of the motor command, and there will therefore be little or no sensory discrepancy resulting from the comparison between the predicted and actual sensory feedback. When the sensory discrepancy is low it is possible to attenuate the effects on sensation induced by self-motion.

An impairment in this 'self-monitoring' mechanism could cause thoughts or actions to become isolated from the sense of will normally associated with them, leading to auditory hallucinations and passivity phenomena that are associated with schizophrenia (Frith, 1992). Frith suggested that these abnormal experiences arise through a lack of awareness of intended actions. The results of the present study support this notion. We demonstrate that patients without auditory hallucinations and passivity experiences and normal subjects perceived selfproduced tactile stimuli as less intense, tickly and pleasant than externally produced tactile stimuli. In contrast, patients with auditory hallucinations and/or passivity experiences showed no difference in their perception of selfproduced and externally produced tactile stimuli. It was the presence of hallucinations and/or passivity experiences, rather than diagnosis, that was associated with this defect in self-monitoring. This suggests that patients who have hallucinations and/or passivity experiences have an abnormal awareness of the sensory consequences of their own movements. These results support the proposal that the experience of passivity arises from a lack of awareness of the predicted limb position based on the forward model (Frith et al. 2000).

From our results it is difficult to assert whether the lack of attenuation of self-produced tactile stimulation in patients with auditory hallucinations and/or passivity phenomena is a state or trait marker. The breakdown in self-monitoring might be considered a state marker because it is possible, if not likely, that some of the patients in the 'Without symptom' group would have had hallucinatory and/or passivity experiences in the past. It is impossible from this study to determine whether people 'With symptoms' at the time of the experiment would continue to show the same results when they are in remission. This is an important question that could be pursued in a future study.

Our results could be interpreted in terms of other sensorimotor gating abnormalities seen in people with schizophrenia. For example, prepulse inhibition, the reduction in startle produced by a prepulse stimulus, is diminished in schizophrenic patients, suggesting that schizophrenia is associated with impaired central inhibitory mechanisms (Grillon et al. 1992; Swerdlow \& Geyer, 1998; Braff et al. 1999). However, although there is evidence that patients with auditory hallucinations and passivity experiences do show reduced prepulse inhibition, deficient prepulse inhibition has been shown to correlate with negative as well as positive symptoms (Braff et al. 1999). It is unlikely that deficient prepulse inhibition accounts for the current results, because the schizophrenic subjects in both groups (with and without auditory hallucinations and/or passivity experiences) in the present study had negative symptoms.

Possible physiological mechanisms by which the attenuation of self-produced tactile stimuli in normal subjects is mediated have been investigated. Neurophysiological data demonstrate that neuronal responses in somatosensory cortex are attenuated by self-generated movement (see Chapman, 1994 for review). For example, active touch is 'gated' in SI of rats (Chapin \& Woodward, 1982) and monkeys (Chapman \& Ageranioti-Belanger, 1991; Jiang et al. 1991) compared to passive and external touch of an identical tactile stimulus. In addition, 
functional imaging (fMRI) data from human subjects demonstrated an increase in activity of the anterior cingulate and somatosensory cortex when subjects experienced an externally produced tactile stimulus on their palm relative to a self-produced tactile stimulus (Blakemore et al. 1998). We propose that this inhibition of anterior cingulate and somatosensory cortex activity by self-generated movements could result from the comparison between predicted and actual sensory feedback, which results in less sensory discrepancy when a tactile stimulus is selfproduced relative to when it is externally produced. There is evidence that this comparison might take place in the cerebellum (Ito, 1970; Paulin et al. 1989; Miall et al. 1993; Leiner et al. 1995; Wolpert et al. 1998), a proposal supported by the functional imaging data (Blakemore et al. 1998). We have suggested that this network of brain areas is involved in predicting the consequences of actions in order to distinguish selfproduced and externally produced sensations. It is possible that this neural system, or part of it, operates abnormally in people with auditory hallucinations and/or passivity experiences.

S.-J.B. and C.D.F. are supported by the Wellcome Trust, E. C.J. and R.S. are employees of and J.S. is a M. Phil, student of the University of Edinburgh. The costs of the work in Edinburgh were met by a grant from the Stanley Foundation.

\section{REFERENCES}

Angel, R. W. \& Malenka, R. C. (1982). Velocity-dependent suppression of cutaneous sensitivity during movement. Experimental Neurology 77, 266-274.

Blakemore, S.-J., Wolpert, D. M. \& Frith, C. D. (1998). Central cancellation of self-produced tickle sensation. Nature Neuroscience 1, 635-640.

Blakemore, S.-J., Frith, C. D. \& Wolpert, D. W. (1999). Spatiotemporal prediction modulates the perception of self-produced stimuli. Journal of Cognitive Neuroscience 11, 551-559.

Braff, D. L., Swerdlow, N. R. \& Geyer, M. A. (1999). Symptom correlates of prepulse inhibition deficits in male schizophrenic patients. American Journal of Psychiatry 156, 596-602.

Chapin, J. K. \& Woodward, D. J. (1982). Somatic sensory transmission to the cortex during movement: gating of single cell responses to touch. Experimental Neurology 78, 654-669.

Chapman, C. E. (1994). Active versus passive touch: factors influencing the transmission of somatosensory signals to primary somatosensory cortex. Canadian Journal of Physiological Pharmacology 72, 558-570

Chapman, C. E. \& Ageranioti-Belanger, S. A. (1991). Comparison of the discharge of primary somatosensory cortical (SI) neurones during active and passive tactile discrimination. Proceedings of the Third IBRO World Congress of Neuroscience, 4-9 August, Montreal, Canada, p. 317.

Chapman, C. E., Bushnell, M. C., Miron, D., Duncan, G. H. \&
Lund, J. P. (1987). Sensory perception during movement in man. Experimental Brain Research 68, 516-524.

Collins, D. F., Cameron, T., Gillard, D. M. \& Prochazka, A. (1998). Muscular sense is attenuated when humans move. Journal of Physiology 508, 635-643.

Decety, J. (1996). Neural representation for action. Reviews in the Neurosciences 7, 285-297.

Frith, C. D. (1992). The Cognitive Neuropsychology of Schizophrenia. LEA: London.

Frith, C. D. \& Done, D. J. (1989). Experiences of alien control in schizophrenia reflect a disorder in the central monitoring of action. Psychological Medicine 19, 359-363.

Frith, C. D., Blakemore, S.-J. \& Wolpert, D. M. (2000). Explaining the symptoms of schizophrenia: abnormalities in the awareness of action. Brain Research Reviews 31, 357-363.

Gallagher, S. (1999). Philosophical conceptions of the self: the implications for cognitive science. Trends in Cognitive Sciences $\mathbf{4}$, $14-21$

Grillon, C., Ameli, R., Charney, D. S., Krystal, J. \& Braff, D. (1992). Startle gating deficits occur across prepulse intensities in schizophrenic patients. Biological Psychiatry 32, 939-943.

Hoffman, R. E. (1986). Verbal hallucinations and language production processes in schizophrenia. Behavioral and Brain Sciences 9, 503-517.

Howell, D. C. (1997). Statistical Methods for Psychology, 4th edn. Duxbury Press: London.

Ito, M. (1970). Neurophysiological aspects of the cerebellar motor control system. International Journal of Neurology 7, 162-176.

Jeannerod, M. (1997). The Cognitive Neuropsychology of Action. Blackwell: Cambridge.

Jiang, W., Chapman, C. E. \& Lamarre, Y. (1991). Modulation of the cutaneous responsiveness of neurones in the primary somatosensory cortex during conditioned arm movements in the monkey. Experimental Brain Research 84, 342-354.

Johnstone, E. C. (1991). Defining characteristics of schizophrenia. British Journal of Psychiatry (Suppl. 13), 5-6.

Kay, S., Fiszbein, A. \& Opler, L. (1987). The positive and negative symptom scale for schizophrenia. Schizophrenia Bulletin 13, 261-276.

Leiner, H. C., Leiner, A. L. \& Dow, R. S. (1995). The underestimated cerebellum. Human Brain Mapping 2, 244-254.

Malenka, R. C., Angel, R. W., Hampton, B. \& Berger, P. A. (1982). Impaired central mismatch error-correcting behavior in schizophrenia. Archives of General Psychiatry 39, 101-107.

Mellors, C. S. (1970). First-rank symptoms of schizophrenia. British Journal of Psychiatry 117, 15-23.

Miall, R. C., Weir, D. J., Wolpert, D. M. \& Stein, J. F. (1993). Is the cerebellum a Smith predictor? Journal of Motor Behaviour 25, 203-216.

Milne, R. J., Aniss, A. M., Kay, N. E. \& Gandevia, S. C. (1988). Reduction in perceived intensity of cutaneous stimuli during movement: a quantitative study. Experimental Brain Research 70, 569-576.

Paulin, M. G. (1989). A Kalman filter theory of the cerebellum. In Dynamic Interactions in Neural Networks: Models and Data, (ed. E. M. A. Arbib and E. S. Amari), pp. 241-259. Springer-Verlag: New York.

Schneider, K. (1959). Clinical Psychopathology. Grune \& Stratton: New York.

Spence, S. A. (1996). Freewill in the light of neuropsychiatry. Philosophy, Psychiatry and Psychology 3, 75-90.

Sperry, R. W. (1950). Neural basis of spontaneous optokinetic responses produced by visual inversion. Journal of Computational Physiological Psychology 43, 482-289.

Swerdlow, N. R. \& Geyer, M. A. (1998). Using an animal model of deficient sensorimotor gating to study the pathophysiology and new treatments of schizophrenia. Schizophrenia Bulletin 24, 285-301.

Von Holst, E. (1954). Relations between the central nervous system and the peripheral organs. British Journal of Animal Behaviour 2, 89-94. 
Weiskrantz, L., Elliot, J. \& Darlington, C. (1971). Preliminary observations of tickling oneself. Nature 230, 598-599.

Wing, J. K., Cooper, J. E. \& Sartorius, N. (1974). Description and Classification of Psychiatric Symptoms. Cambridge University Press: London.

Wolpert, D. M. (1997). Computational approaches to motor control. Trends in Cognitive Sciences 1, 209-216.
Wolpert, D. M., Ghahramani, Z. \& Jordan, M. I. (1995). An internal model for sensorimotor integration. Science 269, 1880-1882.

Wolpert, D. M., Miall, R. C. \& Kawato, M. (1998). Internal models in the cerebellum. Trends in Cognitive Sciences 2, 338-347.

World Health Organization (1992). International Classification of Diseases-10 (ICD-10) Classification of Mental and Behavioural Disorders. WHO: Geneva. 